\title{
Quaderni
}

QUADERNI Communication, technologies, pouvoir

73 | Automne 2010

La métropole parisienne entre récits, paroles et échanges

\section{Ressources numériques pour l'enseignement supérieur, le cas de l'Université en ligne, Laurent Petit}

\section{Christine Barats}

\section{Édition électronique}

URL : http://journals.openedition.org/quaderni/459

DOI : 10.4000/quaderni.459

ISSN : 2105-2956

\section{Éditeur}

Les éditions de la Maison des sciences de l'Homme

\section{Édition imprimée}

Date de publication : 5 octobre 2010

Pagination : 129-132

\section{Référence électronique}

Christine Barats, « Ressources numériques pour l'enseignement supérieur, le cas de l'Université en ligne, Laurent Petit », Quaderni [En ligne], 73 | Automne 2010, mis en ligne le 13 décembre 2012, consulté le 23 septembre 2020. URL : http://journals.openedition.org/quaderni/459 ; DOI : https:// doi.org/10.4000/quaderni.459 


\section{Livre}

ressources

\section{numériques pour}

l'enseignement

supérieur,

le cas de

l'Université

en ligne

\section{Laurent Petit}

Hermès-Lavoisier,

2010

par Christine Barats

Université Paris Descartes
L'Université en ligne (UEL) est un ensemble de ressources numériques destinées aux premiers cycles universitaires dans le domaine des sciences (mathématiques, physique, chimie et biologie). Laurent Petit y a consacré une thèse en sciences de l'information et de la communication, sous la direction de Pierre Moeglin, thèse dont est issu cet ouvrage. Ce projet s'avère exemplaire à double titre : sa longévité (1996-2010) et le nombre de ressources produites (47 modules pour environ 1200 à 2000 heures de formation). Ainsi, indépendamment des changements de noms liés aux différentes phases du projet (Premier cycle sur mesure, Université en ligne, Campusciences et Unisciel), l'Université en ligne a répondu aux différentes politiques ministérielles visant à inciter à la production de ressources pédagogiques pour le supérieur : enseignement sur mesure prônée par Maryse Quéré (1994), opération Campus numérique français $(2000,2001,2002)$ ou plus récemment les Universités Numériques dites Thématiques (UNT). L'inscription de ce projet dans la durée est ainsi une caractéristique relativement rare dans le champ de l'enseignement supérieur, comme le souligne l'auteur. De plus, cet exemple de productions pédagogiques issues d'un collectif d'universitaires constitue un matériel d'investigation original pour examiner les enjeux propres aux technologies éducatives dans un contexte de généralisation des TIC (Technologies de l'Information et de la Communication) et de massification du supérieur. C'est ce que Laurent Petit a entrepris, à partir d'une étude de cas qui s'intéresse aux différentes filiations dans le champ des technologies éducatives (enseignement sur mesure, autoformation...) ainsi qu'à la politique éditoriale. L'auteur fut acteur du projet de 1997 à 2001 pour le compte de la 
sous-direction des technologies de l'information et de la communication pour l'enseignement supérieur ce qui lui a permis de bénéficier de sources difficilement accessibles aux chercheurs. Géographe de formation, il a appliqué la notion de « discordance » à l'analyse de l'UEL afin d'en souligner les contradictions, en particulier dans l'énonciation éditoriale. Son étude est menée à trois niveaux : micro, méso et macro. Le niveau micro est centré sur l'étude en surface des écrans et permet de confronter la politique éditoriale envisagée à celle mise en œuvre afin d'en souligner la relative hétérogénéité. L'analyse des choix éditoriaux des différents écrans (rubriques et contenus) conduit ainsi l'auteur à montrer que l'intention initiale de généralisation est mise à mal par la liberté prise par chacun des auteurs de modules, comme dans le cas d'un polycopié. L'étude de la maquette de l'UEL montre ainsi que la volonté de produire une œuvre collective, comparable à un manuel, achoppe sur la question de la standardisation des méthodes pédagogiques, soulignant les limites des logiques industrielles dans un contexte «d'artisan enseignant». L'hétérogénéité des écrans révèlent ainsi des discordances.

Au niveau méso, l'auteur s'interroge sur les différentes filiations en termes de types d'enseignement envisagés. L'accent porte sur les différentes acceptions de l' « autoformation » dans le champ des technologies éducatives. Il en conclue, que dans le cas de l'UEL, ce terme bénéficie d'approches variées : « autoformation » où l'autonomie de l'apprenant est centrale (autodidaxie) ou au contraire «autoformation" entendue comme renforcement d'une formation en présentiel comparable à la logique d'un polycopié. Cette dernière situation hypothèque une appropriation des ressources produites en dehors des enseignants producteurs et souligne la difficulté de dépasser l'autoproduction et l'autoconsommation qui sont spécifiques à l'enseignement supérieur. Cette étude de cas tend en effet à indiquer que les auteurs des ressources semblent avoir du mal à « concevoir un usage des ressources qu'ils ont produites sans eux. Comment expliquer sinon le fait que l'indexation en éléments fins (une animation, un exercice, etc.), qui permettrait de favoriser les usages les plus ouverts, soit absente du site de référence? » (Petit : 170). En dépit des intentions affichées, «l'utilisateur final correspond donc prioritairement pour un auteur à ses propres étudiants, tout en pensant pouvoir toucher simultanément d'autres publics dans d'autres contextes ». C'est toute l'illusion $\mathrm{du}$ produit universel en autoformation qui est mise au jour. La question des usages, abordée à partir des intentions des concepteurs, confirme la difficulté, dans le champ de l'enseignement, de concevoir et de réaliser des ressources, censées répondre à la tension entre massification des premiers cycles scientifiques et l'attente simultanée d'une individualisation pédagogique.

Le niveau macro consacré aux logiques sociales et modèles socio-économiques éclairent ces tensions et contradictions. Faisant appel aux travaux sur les industries culturelles, l'auteur identifie ainsi plusieurs modèles sous-jacents : principalement le modèle éditorial et le modèle du club. La coexistence de ces deux modèles explique ainsi le caractère disparate de la scénographie. Si le Ministère a souhaité un financement public et privé pour la production de ces ressources - dont la diffusion était pensée au niveau natio- 
nal -, l'étude de cas a montré que, contrairement à l'enseignement secondaire, le financement a été uniquement public. Par ailleurs, la prise en charge par les enseignants des aspects techniques induit une nouvelle division du travail et de nouvelles professionnalisations liées à ces nouvelles compétences. Au-delà des intentions, la technique rend visible les limites des projets d'industrialisation de la formation qui implique une logique de scénarisation. Si un système de conception d'enseignements numériques existe pour le supérieur : Scenari (développé par l'Université Technologique de Compiègne et connu de certains auteurs et concepteurs de l'UEL), la difficulté demeure quant à la réutilisation des ressources en fonction de cadre d'usages multiples (formation initiale, autoformation, formation continue...). La question du numérique, éminemment politique, révèle ainsi de nombreux enjeux : pédagogiques et également d'imposition de normes. Par exemple, la quête de normalisation des standards afin d'indexer les ressources (LOM, SCORM, IMS LD) est un indice de neutralisation de la dimension pédagogique et des contextes d'usage. De plus, l'absence de stabilisation dans ce domaine ne facilite pas leur appropriation par les acteurs concepteurs de ressources, comme ce fut le cas pour l'UEL.

Les ressources de l'UEL conçues à l'origine pour les étudiants des premiers cycles scientifiques universitaires par des enseignants, principalement issus du RUCA ont suscité des tensions qui ont marqué les différentes étapes du projet : tensions liées aux choix pédagogiques, aux types de diffusion... L'ouvrage de Laurent petit ne fait pas l'impasse sur ces aspects que les discours d'accompagnement tendent à gommer pour proposer une vision lisse, voire magique de l'enseignement numérique. Les enseignantsconcepteurs de 1'UEL se sont ainsi opposés quant aux choix de granularisation : découpage des ressources en grains fins (animations, image etc.) ou en gros grains correspondant à des ressources non sécables impliquant une heure ou plus de travail étudiant. Si l'éditeur Dunod ou le cabinet Gartner ont été sollicités pour favoriser une meilleure diffusion de l'UEL et penser les formes de rémunération, il semble que le rôle central de l'enseignant prescripteur ait été mésestimé, de même que le public pour lequel ces ressources ont été pensées, à savoir les premiers cycles universitaires. En effet, si des expériences comparables «trouvent» leur public, il s'agit de publics de cursus sélectifs et non de publics de premiers cycles universitaires. Pour finir, l'auteur, s'inscrivant dans le prolongement des travaux de Bernard Miège ou de Pierre Moeglin envisage une nouvelle fonction afin de faciliter la diffusion de ces ressources et trouver leur public : la fonction de courtage, une intermédiation humaine, connue dans le champ de la formation continue et qui contribue à proposer à une demande, l'offre pertinente correspondante. Cette fonction que Google remplit en partie, ne saurait ici faire l'économie de la dimension humaine, garante de pertinence, confiance et qualité, ce que ne l'on ne peut attendre d'un portail basé sur des algorithmes où la popularité et l'audience $s$ 'avèrent centrales.

L'ensemble de ces questions est au cœur des mutations qui touchent au supérieur et dont témoignent les UNT, comme l'UEL, héritières d'une visée industrialisante dans l'enseignement. Cette étude de cas révèle à la fois les difficultés pour 
penser et conduire un projet dans la durée, tout en s'adaptant aux contextes mouvant du champ des technologies éducatives. Si l'enseignement sur mesure a accompagné l'autoformation, la question demeure entière des conditions d'appropriation des ressources par des enseignants et étudiants chargés de les utiliser. Cet ouvrage souligne ainsi la difficulté de ces attentes et met ainsi au jour les limites du credo de la généralisation des TIC, tout en rappelant les enjeux, dans le champ éducatif, des projets d'industrialisation. 